\title{
Finite temperature dilaton gravity
}

\author{
Zdeněk Kopeckýt \\ Department of Theoretical Physics and Astrophysics, Masaryk University, \\ Kotlářská 2, 61137 Brno, Czech Republic
}

\begin{abstract}
The dilaton free energy density in external static gravitational field is found. We use the real time formulation of the finite temperature field theory and the free energy density is computed to the first order of the string parameter $\alpha^{\prime}$. We obtain the thermal corrections to the $\alpha^{\prime}$ modified Einstein gravity action.
\end{abstract}

PACS numbers: 4.60.+n, 11.17.+y, 05.70.Ce

Typeset using REVTEX 


\section{INTRODUCTION}

The high-temperature properties of quantum gravity are of some interest for their potential cosmological applications. The modifications of the Einstein action have been done in the frame both the finite temperature theory and the string theory.

The finite temperature theory yields the thermal-quantum corrections (by gravitons and others particles) to the effective gravity action [1 4 .

In standard treatments of string theory, it is shown that a consistent string theory can be formulated from some class of conformally invariant models. The $\alpha^{\prime}$ corrections to the Einstein action are known for strings moving in background fields [5],6].

Some aspects of the $\alpha^{\prime}$ modified black hole hermodynamics have been recently investigated in Ref. [7].

In this paper, we derive the dilaton free energy density in curved background which is considered static. The free energy density with opposite sign is contribution to the total gravity Lagrangian. The action for the dilaton field is taken from string theory to order $O\left(\alpha^{\prime}\right)$.

\section{DILATON ACTION}

Corrections to the external metric field action due to stringy effect were carried out to $O\left(\alpha^{\prime}\right)$ in Ref. [6]. The action has form for bosonic string case

$$
S_{0}=\frac{1}{16 \pi} \int d^{D} x \sqrt{-g} e^{-2 \phi}\left(R-4(\nabla \phi)^{2}+\frac{\alpha^{\prime}}{4} R_{k l m n} R^{k l m n}\right)
$$

where $\mathrm{D}=26$ is the dimension corresponds to the critical bosonic string theory, $g_{i k}(x)$ and $\phi(x)$ are the metric tensor field and the dilaton field, respectively. We consider situation without the antisymmetrical background field [5, 6] in the action Eq. (10). The conformal transformation,

$$
g_{i j} \rightarrow \exp \left(\frac{4}{D-2} \phi\right) g_{i j}
$$


together with some field redefinitions, changes the action (11) to [6]

$$
S_{0}=\frac{1}{16 \pi} \int d^{D} x \sqrt{-g}\left(R+\frac{4}{D-2}(\nabla \phi)^{2}+\frac{\alpha^{\prime}}{4} e^{\frac{-4 \phi}{D-2}} R_{k l m n} R^{k l m n}\right) .
$$

We will assume that all but four space-time dimensions are compactified out in the action

(回). In the rest of the article, we consider the four dimensional manifold with signature $(+,-,-,-)$. Related space-time indexes and space indexes have ranges $\mu, \nu, \ldots=0 \ldots 3$ and $i, j, \ldots=1 \ldots 3$, respectively. The dilaton part of the action Eq. (3) we identify with

$$
S[\phi]=\int d^{4} x \sqrt{-g}\left(\frac{1}{8 \pi G}(\nabla \phi)^{2}+\frac{\alpha^{\prime}}{64 \pi G} e^{-2 \phi} R_{\mu \nu \tau \sigma} R^{\mu \nu \tau \sigma}\right) .
$$

We have written out explicitly the gravitational constant in Eq. (四).

\section{DILATON FREE ENERGY TO ORDER $O\left(\alpha^{\prime}\right)$}

In general, statistical mechanics in static space-time can be developed by constructing the following partition function 10,11

$$
Z[\beta]=\operatorname{Tr} \exp (-\beta \hat{H})
$$

The Hamiltonian $\hat{H}$ is connected with $\hat{T}_{0}^{0}$ component of the energy momentum tensor

$$
\hat{H}=\int_{x_{0}=\text { const. }} d^{3} x \sqrt{-g} \hat{T}_{0}^{0} .
$$

The parameter $\beta$ corresponds to the temperature $\beta^{\nu}$ vector 11] in static coordinates

$$
\beta^{\nu}=(\beta, \mathbf{0})
$$

The local Lorentz-rest-frame inverse (scalar) temperature $\beta_{R}$ then is

$$
\beta_{R}=\sqrt{g_{00}} \beta
$$

Now, according to the above construction, we consider dilaton field described by Eq. (丑). The general path integral formulation for the partition function in the Real Time Finite Temperature Theory (RTFT) is 12,13 


$$
\begin{aligned}
Z[\beta] & =\int D \phi \exp (i S[\phi]) \\
& =\exp \left(-\beta \int_{x^{0}=\text { const. }} d^{3} x \sqrt{-g} F[g]\right) .
\end{aligned}
$$

The action $S[\phi]$ is given by Eq. (44). The free energy density $F[g]$ has been introduced in Eq. (9). The time integration $\int d x^{0}$ in the action $S[\phi]$ in (9) is going along curve in complex plane 12,13]. The curve is for the RTFT

$$
\begin{array}{r}
C_{R T F T}=(-t, t) \cup\left(t, t-i \frac{\beta}{2}\right) \cup\left(t-i \frac{\beta}{2},-t-i \frac{\beta}{2}\right) \\
\cup\left(-t-i \frac{\beta}{2},-t-i \beta\right)
\end{array}
$$

and $t \rightarrow \infty$. The integration $\int D \phi$ in (9) is over fields which satisfy the periodicity condition

$$
\phi(-t, \mathbf{x})=\phi(-t-i \beta, \mathbf{x})
$$

at the endpoints of the curve (10).

The interaction part of the action (4) we identify with the second exponential term in Eq. (đ). The free energy density then has the first two terms of the expansion to order $\mathrm{O}\left(\alpha^{\prime}\right)$

$$
F[g]=F_{0}[g]-\frac{\alpha^{\prime}}{64 \pi G} R_{\mu \nu \tau \sigma} R^{\mu \nu \tau \sigma}(V(\beta, g)+1)
$$

where

$$
\begin{aligned}
V(\beta, g) & =\sum_{n=1}^{\infty}(-2)^{2 n} \frac{1}{n !} \frac{1}{2^{n}}\left(i \Delta[\beta]_{11}(x, x)\right)^{n} \\
& =\exp \left(2 i \Delta[\beta]_{11}(x, x)\right)-1
\end{aligned}
$$

The term $F_{0}[g]$ is contribution without the string corrections

$$
F_{0}[g]=-\int d m^{2} \frac{1}{2} \frac{i \Delta[\beta]_{11}(x, x)}{4 \pi G}
$$

The function $\Delta[\beta]_{11}(x, x)$ is the $(1,1)$ component of the RTFT causal Green's function (A8) calculated in the Appendix. We have used the RTFT methods for the computation of the thermal vacuum diagrams [12,13] to evaluate the term (13). The term $V(\beta, g)$ is the sum of the closed connected vacuum graphs, each graph has one vertex with fixed the thermal index 
to 1 12,13 and the vertex is common for $n=1,2,3 \ldots$ number of the thermal propagator function $\Delta[\beta]_{11}(x, x)$ loops. Substitution Eqs. (A8), (A9) in Eq. (14) yields

$$
F_{0}[g]=-\frac{1}{32 \pi^{2}} \int_{0}^{\infty} \frac{d s}{s^{3}} \sum_{k=0}^{\infty} a_{k}(x) s^{k} \sum_{n=-\infty}^{\infty} e^{\frac{-n^{2} \beta_{R}^{2}}{4 s}} .
$$

We have taken limit $m \rightarrow 0$ in Eq. (15). Using analytic continuation relation

$$
\int_{0}^{\infty} d s s^{z} e^{-a s}=\frac{\Gamma(z+1)}{a^{z+1}} .
$$

and the $\zeta$ function regularization [8] we transform Eq. (15) to

$$
\begin{aligned}
F_{0}[g]= & -\frac{1}{(4 \pi)^{2}} \sum_{k=0}^{\infty} a_{k}(x) \Gamma(2-k) \zeta(4-2 k)\left(\frac{2}{\beta_{R}}\right)^{2(2-k)} \\
& -\lim _{\nu \rightarrow 1} \frac{1}{32 \pi^{2}} \frac{a_{2}(x)}{\nu-1}+\frac{1}{64 \pi^{2}} \int_{0}^{\infty} i d s \ln \left(\frac{i s}{8 \pi^{2} G}\right) \frac{\partial^{3}}{\partial(i s)^{3}} \Lambda(x, i s) .
\end{aligned}
$$

The zero temperature term with the pole is canceled against the $\mathrm{k}=2$ term in Eq. (17) because

$$
\lim _{\nu \rightarrow 1} \Gamma(\nu-1) \zeta(0)=\lim _{\nu \rightarrow 1} \frac{-1}{2(\nu-1)}
$$

The $k>2$ terms in Eq. (17) are finite because of the following relation for the product of the $\zeta$ and $\Gamma$ function

$$
\zeta(2 z) \Gamma(z)=4^{z} \pi^{2 z} \frac{\Gamma(1-2 z)}{\Gamma(1-z)} \zeta(1-2 z) .
$$

Final form of Eq. (17) using Eq. (19) will be

$$
\begin{aligned}
F_{0}[g]= & \frac{-\pi^{2}}{90 \beta_{R}^{4}}-a_{1}(x) \frac{1}{24 \beta_{R}^{2}} \\
& -\frac{1}{16 \pi^{2}} \sum_{k=3}^{\infty} a_{k}(x) \frac{(2 k-4) !}{(k-2) !} \zeta(2 k-3)\left(\frac{\beta_{R}}{4 \pi}\right)^{2(k-2)} \\
& +\frac{1}{64 \pi^{2}} \int_{0}^{\infty} i d s \ln \left(\frac{i s}{8 \pi^{2} G}\right) \frac{\partial^{3}}{\partial(i s)^{3}} \Lambda(x, i s) .
\end{aligned}
$$

The Green's function $\Delta[\beta]_{11}(x, x)$ for $m \rightarrow 0$ can be written similarly

$$
\frac{i \Delta[\beta]_{11}(x, x)}{4 \pi G}=\frac{1}{12 \beta_{R}^{2}}+\frac{1}{8 \pi^{2}} \sum_{k=2}^{\infty} a_{k}(x) \frac{(2 k-2) !}{(k-1) !} \zeta(2 k-1)\left(\frac{\beta_{R}}{4 \pi}\right)^{2(k-1)} .
$$


Now we can collect all the terms in Eq. (12) and write final result for the dilaton free energy density

$$
\begin{aligned}
F[g]= & \frac{-\pi^{2}}{90 \beta_{R}^{4}}-a_{1}(x) \frac{1}{24 \beta_{R}^{2}}-\frac{1}{16 \pi^{2}} \sum_{k=3}^{\infty} a_{k}(x) \frac{(2 k-4) !}{(k-2) !} \zeta(2 k-3)\left(\frac{\beta_{R}}{4 \pi}\right)^{2(k-2)} \\
& +\frac{1}{64 \pi^{2}} \int_{0}^{\infty} i d s \ln \left(\frac{i s}{8 \pi^{2} G}\right) \frac{\partial^{3}}{\partial(i s)^{3}} \Lambda(x, i s) \\
& -\frac{\alpha^{\prime}}{64 \pi G} R_{\mu \nu \tau \sigma} R^{\mu \nu \tau \sigma} \exp \left[\frac{2 \pi G}{3 \beta_{R}^{2}}+\frac{G}{\pi} \sum_{k=2}^{\infty} a_{k}(x) \frac{(2 k-2) !}{(k-1) !} \zeta(2 k-1)\left(\frac{\beta_{R}}{4 \pi}\right)^{2(k-1)}\right] .
\end{aligned}
$$

\section{FINITE TEMPERATURE EFFECTIVE $\alpha^{\prime}$ GRAVITY ACTION}

We identify $-F[g]$ with the effective finite temperature Lagrangian and from Eq. (3) we find the modification of the $\alpha^{\prime}$ Einstein action by thermal dilatons

$$
\begin{aligned}
S_{e f f}[g] & =\int d^{4} x \sqrt{-g}\left(\frac{1}{16 \pi G} R-F[g]\right) \\
& =\int d^{4} x \frac{\sqrt{-g}}{16 \pi G\left(\beta_{R}\right)}\left(R-2 \lambda\left(\beta_{R}\right)+\frac{\alpha^{\prime}\left(\beta_{R}\right)}{4} R_{\mu \nu \tau \sigma} R^{\mu \nu \tau \sigma}\right) .
\end{aligned}
$$

We have defined the effective cosmological constant $\lambda\left(\beta_{R}\right)$, the gravitation constant $G\left(\beta_{R}\right)$ and the effective string parameter $\alpha^{\prime}\left(\beta_{R}\right)$ in high temperature limit

$$
\begin{aligned}
& \frac{1}{16 \pi G\left(\beta_{R}\right)}=\frac{1}{16 \pi G}+\frac{1}{6} \frac{1}{24 \beta_{R}^{2}} \\
& \frac{2 \lambda\left(\beta_{R}\right)}{16 \pi G\left(\beta_{R}\right)}=-\frac{\pi^{2}}{90 \beta_{R}^{4}} \\
& \frac{\alpha^{\prime}\left(\beta_{R}\right)}{64 \pi G\left(\beta_{R}\right)}=\frac{\alpha^{\prime}}{64 \pi G} \exp \left(\frac{2 \pi G}{3 \beta_{R}^{2}}\right) .
\end{aligned}
$$

We will introduce the Planck mass $M_{P}=\frac{1}{\sqrt{G}}$ and absolute temperature $T_{R}=\left(\beta_{R}\right)^{-1}$ in Eq. (26) for the effective string parameter $\alpha^{\prime}\left(\beta_{R}\right)$

$$
\alpha^{\prime}\left(\beta_{R}\right)=\alpha^{\prime} \frac{\exp \left(\frac{2 \pi G}{3 \beta_{R}^{2}}\right)}{1+\frac{\pi G}{9 \beta_{R}^{2}}}=\alpha^{\prime} \frac{\exp \left(\frac{2 \pi}{3} \frac{T_{R}^{2}}{M_{P}^{2}}\right)}{1+\frac{\pi}{9} \frac{T_{R}^{2}}{M_{P}^{2}}} .
$$




\section{CONCLUSION}

For the first time, we have derived the free energy density (Eq. (22)) for the dilatons in external static gravitational field using the RTFT methods to order $O\left(\alpha^{\prime}\right)$.

We have found related thermal modification of the $\alpha^{\prime}$ Einstein action. The temperature dependence of the effective string parameter $\alpha^{\prime}\left(\beta_{R}\right)$ in high temperature region is given by Eq. (27). It is clear that the temperature $T_{R}$ has to be comparable with the Planck mass $M_{P}$

to have some observable changes of the effective parameter $\alpha^{\prime}\left(\beta_{R}\right)$. The high temperature behavior of both the effective cosmological constant $\lambda\left(\beta_{R}\right)$ and the gravitational constant $G\left(\beta_{R}\right)$ are similar as in the case of the scalar pregeometry which has been discussed in Ref. [1].

\section{ACKNOWLEDGMENTS}

The author thanks J. Horský, M. Lenc and M. Pardy for discussions. 


\section{APPENDIX: THE REAL TIME GREEN'S FUNCTION}

In this appendix, we will derive the RTFT Green's function for the dilatons without the string corrections $\left(\alpha^{\prime}=0\right.$ in Eq. (4) ) in external static gravitational field.

We can rewrite the first term in the action (四) on the following form

$$
\begin{aligned}
& S[\phi]_{\alpha^{\prime}=0}=-\frac{1}{8 \pi G} \int d^{4} x \sqrt{-g} \phi(x)\left(\square+m^{2}\right) \phi(x) \\
&=-\frac{1}{8 \pi G} \int d^{4} x {\left[\phi(x)\left(\eta^{\mu \nu} \partial_{\mu} \partial_{\nu}+m^{2}\right) \phi(x)\right.} \\
&\left.+\phi(x) E\left(x^{i}, \partial_{x}\right) \phi(x)\right],
\end{aligned}
$$

where

$$
E\left(x^{i}, \partial_{x}\right)=\sqrt{-g}\left(\square+m^{2}\right)-\left(\eta^{\mu \nu} \partial_{\mu} \partial_{\nu}+m^{2}\right)
$$

The second term in Eq. (A2) is calculated with help of the metric $\eta^{\mu \nu}=\operatorname{diag}(1,-1,-1,-1)$. We have introduced helping mass term in the action (凷. We shall take limit $m \rightarrow 0$ in the final equations. It is important that the term $E\left(x^{i}, \partial_{x}\right)$ does not depend on the time variable $x^{0}$ because we consider static space-time.

Now we derive from the RTFT diagrammatic technique [12] RTFT- causal Green's function $\Delta[\beta]\left(x, x^{\prime}\right)$ connected with the action (A1)

$$
\frac{i \Delta[\beta]\left(x, x^{\prime}\right)}{4 \pi G}=\sum_{n=0}^{\infty} i \Delta^{(0)}[\beta]\left(E_{D} i \Delta^{(0)}[\beta]\right)^{n},
$$

where $E_{D}=-\operatorname{diag}\left(E\left(x^{i}, \partial_{x}\right),-E\left(x^{i}, \partial_{x}\right)\right)$ and

$$
i \Delta^{(0)}[\beta](k)=U\left(\beta, k_{0}\right)\left(\begin{array}{cc}
\frac{i}{k^{2}-m^{2}+i \epsilon} & 0 \\
0 & \frac{-i}{k^{2}-m^{2}-i \epsilon}
\end{array}\right) U\left(\beta, k_{0}\right),
$$

where

$$
U\left(\beta, k_{0}\right)=\left(\begin{array}{cc}
\cosh \Theta_{k} & \sinh \Theta_{k} \\
\sinh \Theta_{k} & \cosh \Theta_{k}
\end{array}\right), \cosh ^{2}\left(\Theta_{k}\right)=\frac{1}{1-e^{-\beta\left|k_{0}\right|}} .
$$

Performing the sum (A3) we obtain exact expression for the Green's function

$$
\begin{aligned}
\frac{i \Delta[\beta]\left(x, x^{\prime}\right)}{4 \pi G}=\int & \frac{d k_{0}}{2 \pi} \exp \left(i k_{0}\left(x^{0}-x^{\prime 0}\right)\right) \\
& \times U\left(\beta, k_{0}\right) \operatorname{diag}\left(i \Delta_{F}\left(k_{0}\right)\left(x^{i}, x^{\prime i}\right),\left(i \Delta_{F}\left(k_{0}\right)\left(x^{i}, x^{\prime i}\right)\right)^{*}\right) U\left(\beta, k_{0}\right) .
\end{aligned}
$$


We see that because the external metric field has been considered static it is possible to diagonalize the thermal dilaton Green's function. The propagator $i \Delta_{F}$ is Feynman propagator in curved background which can be expressed in limit $x^{i} \rightarrow x^{i}$ with using relevant DeWitt coefficients $a_{k}(x)$ 8.9]

$$
\begin{aligned}
i \Delta_{F}\left(x, x^{\prime}\right) & =\int \frac{d k_{0}}{2 \pi} e^{i k_{0}\left(x^{0}-x^{\prime 0}\right)} i \Delta[\beta]_{F}\left(k_{0}\right)\left(x^{i}, x^{\prime i}\right) \\
& =\int \frac{d^{4} k}{(2 \pi)^{4}} e^{i k_{0} \sqrt{g 00}\left(x^{0}-x^{\prime 0}\right)} \sum_{n=0}^{\infty} a_{n}(x)\left(-\frac{\partial}{\partial m^{2}}\right)^{n} \frac{i}{k^{2}-m^{2}+i \epsilon} .
\end{aligned}
$$

Now we can express the coincidence limit for $\Delta[\beta]_{11}(x, x)$ using Eqs. (A6), (A7)

$$
\begin{aligned}
& \frac{i \Delta[\beta]_{11}(x, x)}{4 \pi G} \\
& =\int \frac{d^{4} k}{(2 \pi)^{4}} \sum_{n=0}^{\infty} a_{n}(x)\left(-\frac{\partial}{\partial m^{2}}\right)^{n}\left[\frac{i}{k^{2}-m^{2}+i \epsilon}+\frac{2 \pi}{\exp \left(\beta \sqrt{g_{00}}\left|k_{0}\right|\right)-1} \delta\left(k^{2}-m^{2}\right)\right] \\
& =\frac{1}{16 \pi^{2}} \int_{0}^{\infty} \frac{d s}{s^{2}} \exp \left(-s m^{2}\right) \Lambda(x, s) \sum_{n=-\infty}^{\infty} \exp \left(\frac{-\beta_{R}^{2} n^{2}}{4 s}\right)
\end{aligned}
$$

where

$$
\Lambda(x, s) \equiv \sum_{k=0}^{\infty} a_{k}(x) s^{k}
$$

and

$$
\begin{aligned}
& a_{0}(x)=1, a_{1}(x)=\frac{1}{6} R \\
& a_{2}(x)=\frac{1}{72} R^{2}+\frac{1}{180}\left(-R_{\mu \nu} R^{\mu \nu}+R_{\mu \nu \tau \sigma} R^{\mu \nu \tau \sigma}\right)-\frac{1}{30} \square R
\end{aligned}
$$

We have written out explicitly the first three DeWitt coefficients in Eq. (A10). The inverse temperature $\beta_{R}$ is the local Lorenz-rest-frame inverse temperature given by Eq. (8). 


\section{REFERENCES}

$\dagger \quad$ E-mail address: kopecky@elanor.sci.muni.cz

[1] G. Denardo and E. Spallucci, Nuovo Cimento, 69A, 151 (1982).

[2] D. I. Gross, M. J. Perry, and L. G. Yaffe, Phys. Rev. D25, 330 (1982).

[3] P. S. Gribovsky, J. F. Donoghue, and B. R. Holstein, Ann. Phys. (N.Y.) 190, 149 (1989).

[4] A. Rebhan, Nucl. Phys. B351, 706 (1991); ibid B368, 479 (1992).

[5] See for example C. G. Callan, D. Friedan, E. J. Martinec, and M. J. Perry, Nucl. Phys. B262, 593 (1985); M. Green, J. Schwarz, and E. Witten, Superstring Theory, Vol. I (Cambridge University Press, Cambridge, 1987), and references therein.

[6] C. G. Callan, R. C. Myers, and M. J. Perry, Nucl. Phys. B311, 673 (1988).

[7] B. Harms and Y. Leblanc, University of Alabama preprint no. UAHEP-935 and no. UAHEP-936.

[8] N. D. Birrel and P. C. W.Davies, Quantum Fields in Curved Space (Cambridge University Press, Cambridge, 1982), and references therein.

[9] T. S. Bunch and L. Parker, Phys. Rev. D20, 2499 (1979).

[10] J. S. Dowker and G. Kennedy, J. Phys. A11, 895 (1978).

[11] W. Israel, Ann. of Phys. 100, 310 (1978); Physica 106A, 204 (1981).

[12] N. P. Landsman and Ch. G. van Weert, Phys. Rep. 145, 141 (1987).

[13] T. S. Evans, Z. Phys. C-Particles and Fields 36, 153 (1987); ibid 41, 333 (1988). 